\title{
Condiciones laborales de trabajadores sociales en hospitales públicos en la provincia de Mendoza, Argentina. La trastienda de investigación
}

\author{
Cecilia Amalia Molina \\ Licenciada en Trabajo Social. Doctora en Ciencias Sociales \\ Universidad Nacional de Cuyo. Mendoza, Argentina \\ https://orcid.org/0000-0001-5569-786X • ceciliamolina269@gmail.com
}

Yanina Noemi Roslan-Angeloni

Licenciada en Trabajo Social. Especialista en salud pública

Hospital Universitario Mendoza, Argentina.

https://orcid.org/0000-0001-6503-1850 • canyros@yahoo.com.ar

\section{Analía Graciela Correa}

Licenciada en Trabajo Social. Doctora en Ciencias Sociales

Hospital Luis Carlos Lagomaggiore. Mendoza, Argentina

https:// orcid.org/0000-0002-8521-8601 • analiagcorrea@gmail.com

\section{Viviana Elena Varela}

Licenciada en Trabajo Social. Doctora en Ciencias Sociales

Hospital Luis Carlos Lagomaggiore. Mendoza, Argentina

https://orcid.org/0000-0001-9295-3885 • vareladir@yahoo.com.ar

Recibido: 30/01/2020 | Aprobado: 19/05/2020

Esta obra está bajo una Licencia Creative Commons Atribución-NoComercial-CompartirIgual 4.0 Internacional.

Procediencia del artículo: Artículo derivado del proyecto Condiciones de trabajo de los trabajadores sociales que se desempeñan en hospitales públicos del Gran Mendoza. Un análisis a partir del concepto de trabajo decente desarrollado en el periodo 2018-2019. Financiado y aprobado por

el Colegio de profesionales de Trabajo Social de la Provincia de Mendoza, Argentina. 


\section{Resumen}

Este escrito sintetiza resultados de un estudio sobre condiciones de trabajo decente en trabajadores sociales de hospitales públicos de la provincia argentina de Mendoza y detalla los retos que debieron sortearse desde el equipo de investigación por estar sus integrantes involucrados con el problema por indagar.

La metodología es cuanti-cualitativa. Conjuga información obtenida a través de un cuestionario autoadministrado provisto al universo de profesionales de seis hospitales públicos (tasa de respuesta del 75\%) y de entrevistas en profundidad a una muestra intencional que contempló diversidades presentes en el universo.

Como hallazgos significativos, se identifica que las relaciones entre colegas son la vía privilegiada para ingresar a los hospitales y que esas relaciones resultan más confiables para resolver conflictos laborales que los gremios o las autoridades sanitarias. Los consultados dicen gozar de una alta autonomía profesional, pero al mismo tiempo se sienten excluidos de decisiones de política institucional que los afectan laboral y profesionalmente. Consideran que las contribuciones de la disciplina son valoradas por otros miembros de los equipos de salud, pero, en ciertas situaciones, los marcos conceptuales y las propuestas de intervención específicas que conllevan son fuente de destrato.

Se concluye que el concepto de trabajo decente ofrece dimensiones clave para aproximarse a las condiciones laborales de la profesión en diferentes campos. Y que cuando el equipo de investigación es parte del ámbito de estudio, resulta imprescindible ejercitar la reflexividad de manera permanente para producir información rigurosa y a la vez estratégica para el colectivo profesional.

Palabras clave: Trabajo social; Condiciones de trabajo; Trabajo decente; Hospitales públicos; Artesanía intelectual; Reflexividad. 


\title{
Labor Conditions of Social Workers in Public Hospitals in the Province of Mendoza, Argentina. Background of the Investigation
}

\begin{abstract}
This paper summarizes the results of a study on decent work conditions of social workers in public hospitals in Mendoza, Argentina and details the challenges that had to be overcome by the research team as a result of its members being involved in the problem to be investigated.

The methodological design is quantitative and qualitative. It combines information obtained through a self-administered questionnaire provided to professionals from six public hospitals (response rate $75 \%$ ) and in-depth interviews to an intentional sample that contemplated various diversities present in the universe.

Among the significant findings, it is identified that relationships between colleagues are the most privileged way to enter hospitals and that these relationships are more reliable in resolving labor conflicts in comparison with unions or health authorities. Respondents claim to enjoy high professional autonomy, but at the same time, they feel excluded from institutional policy decisions that affect them both in their work and professionally. They consider that the contributions of the discipline are valued by other members of the health teams, but, in certain situations, the theoretical frameworks and the specific proposals that these frameworks entail are a source of abuse.

It concludes that the concept of decent work offers key dimensions to acknowledge the profession's working conditions in different fields. Moreover, when the research team is part of the field of study, it is essential to exercise reflection permanently to produce rigorous and strategic information for the professional team.

Keyworks: Social work; Working conditions; Decent work; Public hospitals; Intellectual crafts; Reflexivity.

Sumario: 1. Introducción, 2. Metodología, 3. Hallazgos 3.1 Oportunidades de empleo y estabilidad en el puesto de trabajo, 3.2 Acceso a la formación profesional para el Trabajo Social hospitalario, 3.3 Remuneración suficiente y trabajo productiva, 3.4 Jornada laboral decente y multiempleo, 3.5 Certezas e incertidumbres en torno a la estabilidad 3.6 Trato justo y destratos en el trabajo 3.7 Las disputas entre modelos de atención como fuente de destratos interprofesionales 3.8 Límites y posibilidades de autonomía profesional, 3.9 Riesgos, conflictos y respuestas para enfrentarlos en los espacios laborales 3.10 Diálogo social y relaciones laborales, 4 . Conclusiones, 5. Referencias bibliográficas.
\end{abstract}




\section{Introducción}

Este escrito recupera parte de un análisis sobre condiciones de trabajo de las y los trabajadores sociales que se desempeñan en hospitales públicos de la provincia de Mendoza, Argentina, a la luz de la categoría trabajo decente, que fue acuñada por la Organización Internacional del Trabajo (OIT) en 1999, cuando comenzaron a quedar al desnudo los graves efectos de las recetas neoliberales aplicadas a nivel global. En sintonía con el paradigma de los derechos humanos, el trabajo decente contempla las condiciones de libertad, equidad, seguridad y dignidad en las que se ejerce el trabajo, además de remuneraciones adecuadas a las tareas que se realizan (Somavía, 2014) y se vincula con la aspiración de avanzar hacia una sociedad más justa y productiva (Gálvez-Santillán, 2016).

El segundo propósito del escrito es detallar las vicisitudes que se transitaron en el diseño metodológico y en el trabajo de campo, esto es: dar cuenta de la cocina de la investigación (De Sousa-Minayo, 2009) y de cómo se ejercitó la reflexividad, en la línea que proponen Bourdieu y Wacquant (2008).

Entendemos que explicitar y compartir los secretos de los procedimientos seguidos y el modo en que las propias posiciones y decisiones de los investigadores dan forma a las conclusiones fAmezcua y Gálvez, 2002; Gilson, 2012; Salas-Zapata, Ríos-Osorio, GómezArias y Álvarez Del Castillo, 2012), puede contribuir a identificar retos potenciales por sortear cuando equipo y población de estudio comparten el ámbito de análisis (los servicios sociales hospitalarios en este caso), la misma categoría profesional, y las condiciones de trabajo (el problema a estudiar).

A contramano de la noción conservadora que se aproxima a los trabajadores como fuerza de trabajo y que se focaliza en las normas de higiene y seguridad laboral, en los riesgos individuales y en las respuestas especializadas a esos riesgos, Neffa (1995) propone entender al trabajador como parte fundamental del proceso de trabajo. Desde esta perspectiva crítica, condiciones y medio ambiente de trabajo son claves, y la salud de los trabajadores es un asunto colectivo que como tal demanda soluciones también colectivas. Refiriéndose particularmente al trabajo en salud, el mismo autor señala:

El trabajo también implica una movilización de los recursos psíquicos y mentales, pues para lograr el objetivo de prestar el servicio de cuidado y curación, se requiere disponer de conocimientos, haber acumulado experiencia, captar y procesar información, mostrar interés y estar motivado e involucrado, tanto a nivel individual como en relación con el colectivo de trabajo. Pero esta complejidad del trabajo está negada, y una parte de la actividad está invisibilizada y subestimada. Por eso, generalmente el trabajo no se recompensa ni se reconoce adecuadamente. (Neffa y Henry, 2017, pp. 18-19). 
Molina, Roslan-Angeloni, Correa y Varela

La categoría trabajo decente que orienta el estudio sintoniza con la concepción renovadora del trabajo que defiende Neffa. “Ambas entienden al trabajador como sujeto que produce y para quien se produce; el trabajador es quien trabaja y quien debe gozar del fruto de su trabajo" (Sánchez, 2010, p. 70). Los trabajadores, para esta óptica, no son fuerza ni un recurso al servicio de un resultado.

Los trabajadores del sector salud, entre ellos los trabajadores sociales, lidian a diario con los resabios de las políticas sanitarias pro mercado en las organizaciones sanitarias. Esas políticas, adoptadas por los gobiernos de la mayoría de los países latinoamericanos en las últimas décadas del siglo pasado, tuvieron cabida de la mano de profundas reformas en las relaciones de trabajo, mediante cambios en las regulaciones que traspasaron al sector salud (Brito-Quintana, 2000).

Sin desconocer los avances que supone incorporar en la jerga de la política laboral la idea de trabajo decente ${ }^{1}$ y los logros de políticas sociales implementadas por gobiernos sociales de derecho en el continente, en la primera década del presente siglo (Laurell, 2014),los trabajadores del sector salud no son ajenos a los efectos del neoliberalismo sanitario.

El proyecto político neoliberal introdujo criterios de evaluación económica de las prácticas e instrumentos de estandarización de actividades que desafiaron la autonomía profesional (Molina y Tobar, 2018), ajustaron los procesos de trabajo de las profesiones de salud en general al tiempo que expusieron a los trabajadores a mecanismos que flexibilizan la estabilidad laboral (Homedes \& Ugalde, 2005; Rovere y Abramzón, 2005), contrariando las aspiraciones del trabajo decente en salud.

Al deteriorar las condiciones laborales de los equipos de atención e incrementar la demanda sobre los servicios públicos de salud, las reformas y sus efectos persistentes

Repercuten negativamente en la calidad de la asistencia, en la capacidad de prevenir enfermedades, en el desempeño de los sistemas de salud y, en definitiva, en el logro de los resultados de salud deseados, como los señalados en los Objetivos de Desarrollo del Milenio. (Sánchez, 2010, p. 10).

1 En Argentina, durante la última década, el trabajo decente ha sido definido como una prioridad pública. Desde entonces “el Ministerio de Trabajo, Empleo y Seguridad Social debe promover la inclusión del concepto en las políticas nacionales, provinciales y municipales” (Acevedo, Farías, Sánchez, Astegiano, Fernández, 2012, p. 17). 
Este panorama ha dado pie a investigaciones orientadas por el concepto de trabajo decente focalizadas en enfermeros (Aspiazu, 2016, 2017; Quintana-Zavala, ValenzuelaSuazo y Paravic-Klijn, 2014), en médicos hospitalarios (Gálvez-Santillán, 2016; Lanari, 2010) y en equipos de salud del primer nivel de atención (Acevedo, Farías, Sánchez, Astegiano y Fernández, 2012). Todas concluyen que las condiciones en las que trabajan las diferentes disciplinas relacionadas con la salud impactan de manera directa en la dignidad en el empleo y en la calidad de la atención que se brinda a la población. A tal punto hay certeza de que esos tópicos están vinculados, que se afirma que no es posible "prevenir ni atender adecuadamente las necesidades de la salud si no se cuenta con el recurso humano calificado, bien remunerado y motivado para la prestación del servicio" (Burijovich y Pautassi citados en Sánchez, 2010, p. 11).

La falta de estudios locales que analicen la situación laboral del colectivo de trabajadores sociales hospitalarios, apelando a la categoría trabajo decente en sus distintas dimensiones, dio pie a una investigación que fue avalada por el Colegio de Trabajadores Sociales de Mendoza, por considerarla estratégica en la actual coyuntura histórica del continente, el país y la profesión.

Los objetivos de la indagación fueron tres: El primero, identificar las condiciones de trabajo decente de los trabajadores sociales atendiendo a dimensiones propuestas por Somavía (2014), como acceso al empleo de calidad y en cantidad suficiente, ingresos adecuados, seguridad, formación profesional continua y pertinente a la empleabilidad; respeto de los derechos, fortalecimiento sindical y negociación colectiva, participación en las decisiones de política económica y social, diálogo social y protección social en el empleo en condiciones de libertad, equidad y dignidad. El segundo objetivo consistió en identificar posibles diferencias en las dimensiones antes mencionadas según el tipo de inserción laboral de los profesionales y tipo de hospital en el que trabajan. Y el tercer objetivo fue explorar las potencialidades y límites del concepto de trabajo decente y sus dimensiones para su aplicación en ámbitos de inserción disciplinar diferentes del hospitalario.

El presente escrito, en el que se expone una parte de los resultados de la investigación, está organizado en tres apartados. En primer lugar, se expone, justifica y reflexiona acerca del diseño metodológico adoptado y las adaptaciones que el equipo responsable del estudio realizó sobre la marcha para alcanzar los objetivos antes enunciados. A continuación, se sintetiza parte de los resultados, organizándolos según algunas de las dimensiones del trabajo decente propuestas por la OIT, y se cierra con conclusiones. 


\section{Metodología}

Se optó por un diseño que combinó técnicas cuantitativas y cualitativas para triangular resultados, atenuar las limitaciones que las técnicas tienen por separadoy valernos de las ventajas complementarias de cada una para lograr mayor exactitud (Vasilachis, 1992).

Las anticipaciones de sentido que orientaron el estudio fueron dos. Se sostuvo que los trabajadores sociales que se desempeñan en hospitales públicos conviven con situaciones donde se respeta la igualdad de oportunidades y trato, sin tener del todo garantizados los derechos a la organización y participación en las decisiones de política institucional que afectan su tarea. Se sostuvo también que no hay grandes diferencias en las condiciones laborales de los profesionales en los hospitales, aun cuando se vinculan con poblaciones y problemáticas diferentes y los lugares en los que trabajan tienen diferente capacidad de resolución.

Seis hospitales públicos del Gran Mendoza constituyeron el ámbito del estudio, tres de los cuales son centros de referencia para toda la provincia y otros tres son de alcance regional ${ }^{2}$.

En cuanto a las unidades de análisis, el estudio procuró abordar el universo de los trabajadores sociales $(55)^{3}$ que se desempeñan bajo diferentes modalidades de inserción laboral: como personal de planta permanente, contratados, cursando residencias de posgrado ${ }^{4}$, integrantes del servicio social del hospital o profesionales que están insertos en los centros para ejecutar programas focalizados nacionales y provinciales, como el programa de sida, el programa oncológico, el de salud sexual y reproductiva y el de malformaciones congénitas.

El componente cuantitativo del estudio fue efectivamente respondido por 41 profesionales, quienes representaron el $75 \%$ de la población por estudiar. Para asegurar una tasa aceptable de respuestas, se realizaron reuniones previas presenciales con integrantes de los servicios, en las que se explicaron los alcances del estudio y se salvaron dudas emergentes. En el transcurso de esos encuentros, algunos colegas manifestaron

2 Se trata de los hospitales de alta complejidad: Luis Lagomaggiore (principal maternidad de Mendoza, con más de 6.000 partos por año), Central (que maneja la accidentología compleja y los trasplantes), el pediátrico Humberto Notti y los hospitales de segundo nivel de complejidad Ramón Carrillo, Diego Paroissiens y José Néstor Lencinas. Se excluyeron del estudio los dos hospitales de salud mental por entender que presentan especificidades que excedían las pretensiones del estudio.

3 Excluidos cuatro de los miembros del equipo de investigación.

4 Las residencias de Trabajo social en salud pública y de Trabajo social en salud mental. 
reticencias y temores de expresar abiertamente sus opiniones sobre el tópico por analizar. Los menos, pusieron de por medio objeciones a la política del Colegio Profesional que respaldaba el estudio. Estas situaciones alertaron al equipo de investigación sobre la necesidad de garantizar confidencialidad, por lo cual se decidió eliminar algunas variables de identificación, como años de antigüedad en el puesto o formación de posgrado, a sabiendas de que esa decisión podía afectar las comparaciones que se plantearon como uno de los objetivos específicos del estudio. Esto es: se optó por resignar ${ }^{5}$ información que pudiera dar cuenta de los perfiles de los participantes y asociarla con el tipo de respuestas, para garantizar mayor participación.

El componente cualitativo, por su parte, se trabajó a partir de una muestra intencional.

La construcción de los instrumentos de recolección de datos (cuestionario autoadministrado y entrevistas en profundidad) se realizó a partir de operacionalizar el concepto de trabajo decente en base a las dimensiones desarrolladas por la OIT $^{6}$ y a decisiones del equipo relacionadas con ciertas especificidades del Trabajo Social que interesó conocer.

El cuestionario tomó la forma de un formulario electrónico y fue enviado por correo electrónico en mayo de 2018 a quienes manifestaron su decisión de participar después de encuentros presenciales y/o contactos telefónicos. En el cuerpo del correo se incluyeron una breve descripción de la investigación y una solicitud de consentimiento informado que se consideró aceptada con el llenado del formulario. Los cuestionarios autoadministrados fueron anónimos y debían contestarse por correo electrónico en plazos acordados entre participantes y equipo.

El cuestionario estuvo compuesto por 33 preguntas, la mayor parte con opciones predefinidas de respuesta, distribuidas en 6 bloques correspondientes a las dimensiones del trabajo decente, según la operacionalización realizada por el equipo de investigación. En todos los casos, cuando la posible respuesta correspondía a una categoría no definida ("otro/a"), se abrió un campo para su explicitación por parte del encuestado. Se agregó un último campo para comentarios libres y se preguntaron datos de base (edad y sexo).

5 Omitir

6 Los indicadores para la medición del trabajo decente según la OIT son: oportunidades de empleo, remuneración suficiente y trabajo productivo, jornada laboral decente, estabilidad y seguridad del empleo, trato justo en el trabajo, seguridad en el trabajo (trabajo seguro), diálogo social y relaciones laborales (Somavía, 2014). Anker, Chernyshev, Egger, Mehran y Ritter (2003) sugieren incorporar como indicador específico para indagar en la estabilidad laboral la percepción de la estabilidad del empleo futura. Esta cuestión también fue contemplada en el cuestionario. 
Molina, Roslan-Angeloni, Correa y Varela

Por las características de la herramienta con la cual se realizó el cuestionario, la confidencialidad de las respuestas quedó totalmente garantizada, ya que la plataforma Google utilizada no permite identificar la dirección de correo electrónico desde la cual se responde el formulario, figurando solamente día y hora del llenado del mismo.

Antes de la aplicación definitiva del cuestionario, se realizó una prueba piloto con profesionales de los hospitales de la aseguradora de salud de los empleados públicos de Mendoza. ${ }^{7}$ La opción para probar el instrumento de recolección de datos con ese colectivo obedeció a que se trata de profesionales que se desempeñan en centros asistenciales de la seguridad social, que en el contexto provincial guardan cierta semejanza con los hospitales de propiedad estatal.

Se confeccionó el cuestionario para que pudiera ser resuelto en no más de 10 minutos atendiendo a la necesidad de procurar agilidad y una tasa de respuesta aceptable. Inicialmente estimamos obtener los resultados en dos semanas para continuar con el momento cualitativo, a través de los grupos de discusión, a finales del mismo mes. Como el nivel de respuestas no fue el esperado se debieron implementar una serie de estrategias de recordación (llamadas telefónicas, mensajes de texto, reenvío de mails, visitas personales a los y las colegas en sus lugares de trabajo) para incentivar el llenado del cuestionario. Estas tareas no habían sido contempladas en el cronograma de actividades e insumieron tiempos y esfuerzos imprevistos para el equipo de investigación. Implicaron un desafío y obligaron a aplazar la segunda etapa del trabajo de campo dos meses después de lo originalmente planificado.

Con el componente cualitativo del estudio se pretendió profundizar en la información obtenida a través de los cuestionarios, incorporar nuevas descripciones y explicaciones y testear las hipótesis provisorias que el equipo fue elaborando a partir de los resultados cuantitativos. Esta instancia de la investigación se concretó a través de cuatro entrevistas en profundidad a trabajadores sociales que habían respondido el cuestionario.

Los entrevistados fueron tres mujeres y un varón que trabajan en hospitales de alta y mediana complejidad. La relación laboral de los entrevistados con el hospital fue variada: una fue una empleada de planta a punto de jubilarse; otra, una residente de Trabajo Social; una profesional contratada desde un programa provincial con base operativa en un hospital, y un trabajador social empleado de planta con cinco años de antigüedad. El análisis se realizó agrupando categorías alrededor de las hipótesis y líneas de análisis definidas por el equipo previamente.

7 Hospital El Carmen, Hospital Fleming y Hospital Virgen de la Misericordia. 
En lugar de entrevistas, el diseño original del proyecto contemplaba profundizar en los resultados del cuestionario a través de uno o dos grupos de discusión. El reemplazo de esa técnica por las entrevistas en profundidad fue, en parte, una respuesta al miedo puesto en palabras por potenciales participantes de que sus jefes o los propios compañeros conocieran las respuestas.

Frente a estos reparos, el equipo de investigación acordó poner especial cuidado en despejar prevenciones sobre el tratamiento y los destinatarios de la información, así como sobre el papel del Colegio de Trabajadores Sociales y de las investigadoras en el proceso. Por ese mismo motivo - preservar la confidencialidad de los entrevistados y resguardar la objetividad en la aplicación de la técnica-, se decidió delegar también en un investigador ajeno al ámbito de estudio y a la Disciplina la administración de la base de datos y el análisis preliminar de la información.

Este viraje también fue decidido por el equipo a raíz de los inconvenientes acontecidos en la primera etapa, antes descritos. Por lo tanto, se priorizó profundizar los resultados de los cuestionarios mediante entrevistas en profundidad a colegas que habían mostrado interés en el tema frente a otros cuya eventual participación implicaba una dilación de los tiempos del trabajo de campo, además de incertidumbre por la posible no respuesta.

Las entrevistas estuvieron a cargo de una socióloga externa al equipo, se realizaron en lugares elegidos por los participantes, entre agosto y setiembre de 2018, y tuvieron una duración promedio de 45 minutos cada una. Fueron grabadas con el consentimiento de los entrevistados y desgrabadas ${ }^{8}$ en su totalidad.

El análisis de la información obtenida se realizó agrupando categorías alrededor de los supuestos y de líneas de análisis previamente definidas por el equipo. El método de análisis de datos utilizado para la interpretación fue el análisis comprensivo.

La inquietud por compartir las decisiones metodológicas que se tomaron durante la marcha del estudio y de exponerlas como se ha tratado está en sintonía con un requisito de la perspectiva cualitativa, de acuerdo con la cual:

Los resultados de una investigación dependen del conjunto de elecciones que el investigador/metodólogo ha realizado a lo largo de todo el recorrido. Por este motivo, en el informe de investigación se debe dedicar amplio espacio para detallar y comentar las elecciones hechas, evaluando en lo posible sus impactos sobre los resultados (Marradi, 2002, p. 119).

\footnotetext{
${ }^{8}$ Transcritas.
} 


\section{Hallazgos}

\subsection{Oportunidades de empleo y estabilidad en el puesto de trabajo}

Las respuestas de los cuestionarios arrojaron que las oportunidades para ingresar a trabajar en hospitales tienen distintas formas posibles. Las redes entre colegas emergieron como la vía privilegiada para comenzar a trabajar.Solo 1 de cada 4 encuestados ingresó por concurso, mientras que casi la mitad lo hizo a partir de la recomendación de colegas u otros contactos personales o académicos. Otra modalidad de ingreso referida en las entrevistas fue la reasignación de funciones desde otras áreas de política social hacia el sector salud, como resultado de las reestructuraciones que se producen en el Estado.

Estaba en el Instituto de la vivienda con muchísimos trabajadores sociales y cuando a mediados de los 90 se descentralizó la política en los municipios, nos dieron opciones (...) yo fui primero al Plan materno-infantil del Ministerio de Salud, ahí pidieron trabajadores sociales para este centro y así llegué. (TS de planta permanente, hospital de alta complejidad).

A la pregunta sobre a qué motivaciones obedece trabajar en un hospital público, lo primero que se esgrime es el gusto o interés por el campo sanitario (35\%). La interdisciplina, la complejidad de las situaciones que se abordan, la capacitación permanente $\mathrm{y}$, finalmente, la gratificación que resulta de contribuir al bienestar de los usuarios son otras motivaciones que hacen que los profesionales elijan ingresar y/o permanecer en este ámbito laboral. En general sienten que es un campo "desafiante" y "muy dinámico". Al mismo tiempo, ponen en valor la seguridad que supone trabajar "puertas adentro" de una organización. "La institución, te da un techo, te da un sostén, y trabajo estable... no te exponés tanto a situaciones de peligro como otras colegas" (TS residente, hospital de alta complejidad).

En cuanto al tipo de relación laboral que vincula a los profesionales con la organización empleadora, posibilitando mayor o menor estabilidad, la mitad de los consultados pertenece a planta permanente del hospital; $20 \%$ se encuentra haciendo la residencia de Trabajo Social y un porcentaje similar integra los equipos de programas del Ministerio de Salud. El 5\% restante son contratados de locación de servicios.

Residentes y contratados, quienes no forman parte de la planta permanente (25\%) se consideran en "situación de subempleo", ya que su vínculo laboral es inestable. Y, en algunos casos, expresan, como advirtieron Acevedo et al. (2012), que la diversidad de condiciones de trabajo en cuanto a estabilidad laboral y protección social con las que se 
convive genera inequidades entre personas que realizan labores semejantes, lo cual es causa de malestar.

\subsection{Acceso a la formación profesional para el Trabajo Social hospitalario}

El Trabajo Social en hospitales emerge como un campo que fuerza a capacitarse, aun cuando la capacitación no aparezca como condición para preservar el empleo. Al indagar sobre la formación profesional a través de los cuestionarios, $80 \%$ de quienes respondieron dijeron haber combinado distintas instancias de formación de posgrado en más de una oportunidad, en muchos casos por iniciativa propia.

\subsection{Remuneración suficiente y productiva}

Según los cuestionarios, 1 de cada 4 encuestados considera que la remuneración que percibe por sus tareas es suficiente y algo más de la mitad valora que es insuficiente ${ }^{9}$. Los argumentos más frecuentes entre quienes están disconformes con su remuneración tienen que ver con la valoración de la responsabilidad y los riesgos que implica el trabajo hospitalario. Aducen, como lo marca la literatura, que están involucrados en una actividad que debe diferenciarse del resto, por ser "de mano de obra intensiva, prestar un servicio de alta repercusión social y por poseer recursos humanos altamente calificados" (Novick y Galín citados en Aspiazu, 2016, p. 57).

Profundizado este tópico en las entrevistas, emerge que la valoración de los ingresos como suficientes remite a la comparación de los salarios con los de colegas que trabajan en otros ámbitos de la política pública donde, con la excepción de quienes se desempeñan en la Justicia, los ingresos son significativamente menores que los de Salud. En concreto, se pondera el hecho de que la carrera de Trabajo Social en salud esté sujeta al régimen médico como un factor que categoriza el salario en el sector.

\subsection{Jornada laboral decente y multiempleo}

Tres de cada cuatro consultados trabaja bajo el régimen de 24 horas semanales, mientras que $17 \%$ tiene casi el doble de carga horaria (entre 44 y 49 horas por semana). La mayor parte de este último segmento está constituida por residentes.

Un tercio de los encuestados que trabaja 24 horas en el hospital suma mínimo otras 10 horas semanales en un segundo empleo, en general, en otro organismo público. El

9 La escala de respuestas contemplaba las opciones muy insuficiente, insuficiente, suficiente y más que suficiente. 
Molina, Roslan-Angeloni, Correa y Varela

máximo de horas trabajadas por semana de grupo son entre el trabajo hospitalario y el que se realiza afuera.

Ahora bien, no necesariamente el multiempleo obedece a razones económicas. Algunos colegas consideran la diversificación del empleo como una estrategia de protección de su salud mental, y otros, como una oportunidad para desplegar diferentes capacidades. "En la tarde doy clases. No lo hago por necesidad económica, es una cuestión de vocación, me gusta, me conecta con otro espacio" (TS de planta permanente, hospital de alta complejidad).

El panorama observado en los hospitales del Gran Mendoza respecto de este punto se asemeja al trazado en un estudio previo de alcance nacional sobre la situación de la fuerza laboral del sector salud de Argentina bajo la perspectiva del trabajo decente. Advierte Sánchez (2010) que el pluriempleo es mayor en salud que en otros sectores de la economía, aun cuando los salarios medios del sector no distan demasiado de los salarios del resto.

Por otra parte, ya sea que mantengan o no trabajos distintos al del hospital, la alta feminización de la profesión en el ámbito hospitalario y la función social vinculada a tareas de cuidado que se le atribuye hacen que buena parte de las entrevistadas expresen cautela al momento de equilibrar la extensión de la jornada laboral con las tareas familiares.

Quedás muy cansada, y no me parece apropiado estar más horas (...). Yo he tratado de ir armonizando los tiempos de trabajo con las obligaciones personales, la edad de tus hijos. Uno se va adaptando en el camino. Yo tengo 30 años de antigüedad y entonces, bueno, al principio no tenía tantas horas, tenía uno solo y más chicos, ahora que mis hijos están más grandes trabajo más tiempo. (TS de planta permanente, hospital de alta complejidad).

\subsection{Certezas e incertidumbres en torno a la estabilidad}

El $80 \%$ de los encuestados considera que su trabajo es estable, lo cual supone remuneración por antigüedad, derecho a vacaciones y aportes jubilatorios. Dentro del 20\% restante, que considera que está en situación de inestabilidad, están los trabajadores cuyo vínculo laboral con el hospital es a través de residencias y/o contratos de locación de servicios.

Si bien la mayoría de los consultados posee trabajo estable, la percepción de la estabilidad futura es más pobre.El $40 \%$ de los profesionales manifiesta sentir incertidumbre o temor respecto de posibles cambios negativos en la situación laboral. En 
este grupo prevalecen trabajadores sociales en planta permanente, por lo cual la percepción pesimista no tendría vínculo directo con la estabilidad laboral individual. Los cambios en los presupuestos de los programas y las dudas sobre la permanencia de los mismos con los cambios de gobierno, las constantes reorganizaciones de la política sanitaria y las reformas previsionales son los argumentos que alimentan la incertidumbre laboral. Entran en consideración también factores contextuales relacionados con las transformaciones neoliberales de la economía, del mercado de trabajo y del sector salud.

La incertidumbre se asocia a una percepción compartida de acuerdo con la cual, cuando hay recortes en el sector salud, se prioriza a los médicos sobre el resto de los profesionales quienes corren más riesgos de ser desvinculados. De este temor se han hecho eco otros estudios.

A pesar de los cambios, los médicos, por la propia jerarquía que les otorgan sus conocimientos, mantienen una posición hegemónica que propicia asimetrías con el resto de los trabajadores no médicos. Esa posición los haría susceptibles de realizar trabajos con niveles de menor déficit de TD que los otros trabajadores de salud. (Lanari, 2010, p. 35).

\subsection{Trato justo y destrato ${ }^{10}$ en el trabajo}

Dos de cada tres respondentes aseguraron que en oportunidades habían sufrido trato discriminatorio dentro del hospital, aunque la mayor parte de ellos lo consideró excepcional. La causa más frecuentemente atribuida a esta situación fue la "propia profesión" (40\%), seguida por las diferencias en las formaciones académicas con otros profesionales, en especial los médicos, $(21 \%)$, la ideología política $(15 \%)$ y el género $(11 \%)$.

La primera causal de discriminación aquí marcada puede relacionarse con lo planteado por Neffa y Henry (2017, p. 391), para quienes "el alto nivel de profesionalización que caracteriza a las organizaciones de salud (...) habilita determinadas relaciones interpersonales que, en muchos casos, se basan en relaciones de poder asociadas a representaciones asimétricas en torno a los distintos saberes".

Consultados acerca de la conducta que siguió al trato discriminatorio, $90 \%$ de quienes respondieron al cuestionario dijeron haberse sentido discriminados o maltratados no informaron ni denunciaron formalmente la situación ante ninguna autoridad, aun cuando identificaban canales institucionales para el planteamiento y resolución de conflictos. De hecho, la mayoría de este segmento opinó que, en caso de tener un problema laboral, sería fácil expresarlo y obtener una solución justa en la organización.

10 Maltrato 


\subsection{Las disputas entre modelos de atención como fuente de destratos interprofesionales}

Cuando se indaga sobre las situaciones que desencadenan malos tratos o discriminación desde otras disciplinas o las autoridades hacia los trabajadores sociales del hospital, los participantes del estudio coinciden en que la mayoría de esas situaciones ocurren por discrepancias en los ritmos esperados de respuesta a los problemas. Por lo general, la intervención profesional implica gestionar procesos mediadores, asesorar a familias y articular respuestas con otras instituciones de política social. Y estas tareas suponen tiempos que no siempre coinciden con la expectativa del modelo de atención dominante, que omite los condicionamientos sociopolíticos del proceso salud-enfermedad y las subjetividades de los involucrados, componentes clave de la intervención de Trabajo Social en salud. Según los entrevistados, difícilmente esta complejidad es asumida por el resto del equipo de profesionales médicos y entonces la demanda es "habilitar rápido las camas luego de compensar los cuadro clínicos". Los conflictos y malestares en el trabajo se hacen patentes cuando no se responde en los tiempos que pretende el modelo, si bien los trabajadores sociales generan estrategias para negociar, para imponer el criterio técnico y construir consensos con los equipos médicos, se sienten desgastados frente a este "otro trabajo constante".

La urgencia me gusta, me he acostumbrado, pero reconozco que hay momentos en los que siento que estaría bueno estar en un espacio donde la urgencia no te persiga tanto (...) nunca dejamos nublar la intervención o que se hagan cosas que no corresponden, y si este paciente se tiene que quedar para que se lo reevalúe, se quedará, contra viento y marea, se quedará. Pero esto cansa. (TS residente, hospital de alta complejidad).

Se observa que las perspectivas teóricas en disputa en el campo de la salud y las valoraciones diferenciadas sobre los saberes especializados que portan los diferentes integrantes de los equipos de salud son vividas como límites para el trabajo decente. Y que, si bien se han logrado avances en el reconocimiento de las funciones del Trabajo Social en hospitales, esas "luchas de poder, de carácter simbólico-político con resultados prácticos (...) varían según cómo se ubique la profesión y las/os profesionales en cada contexto político e institucional" (Nucci, Crosetto, Bilavcik y Miani, 2018, p. 13).

Las intervenciones que logran cuestionar las representaciones sociales instaladas en los hospitales, y habilitan transitar los discursos y las prácticas hegemónicas, ubicando desde otro lugar el Trabajo Social, generan un desgaste laboral. Siguiendo a Cazzaniga (2007, p. 109), “la capacidad de argumentar, la rigurosidad teórica y la intervención responsable posicionan de otra manera al Trabajo Social y otorgan condiciones para el ejercicio de poder, en este caso poder decir, poder hacer, poder construir". Para los consultados en el estudio, sin embargo, ejercitar ese poder alimenta discrepancias y reparos que se 
Molina, Roslan-Angeloni, Correa y Varela

asocian a trato injusto en el trabajo, siendo los propios pares, compañeros de equipos de salud, especialmente médicos y médicas, los principales dispensadores de destrato.

\subsection{Límites y posibilidades de autonomía profesional}

La gran mayoría de los encuestados considera que goza de un grado considerable de autonomía en el trabajo diario, entendiendo a esta como la libertad para aplicar el conocimiento especializado de la profesión, empleando el criterio técnico y el saber propio en la toma de decisiones. En una escala del 1 al 10 (donde 1 significa autonomía nula y 10 autonomía absoluta), el 80\% de los encuestados ubicó su desempeño en el hospital entre los puntos 8, 9 y 10. Pero, como se mencionó antes, los criterios de la profesión confrontan con criterios biomédicos y/o con las normas institucionales, que tienden a ser más rígidas o punitivas con los sujetos.

Con frecuencia, los respondentes sienten que se espera que los trabajadores sociales ejerzan una especie de "función de policía" sobre los pacientes o sus familias y que moralicen o "normalicen" conductas y relaciones.

La médica nos decía: "tienen que llamar a la familia porque esa familia no viene, mándenla al Juzgado, llamen a la policía para que vaya a la casa y los traiga". La verdad es que nosotros no nos podemos quedar pegados a eso. Claro, "doctora, mire, disculpe, pero eso nosotros no lo vamos a hacer, no necesitamos llegar hasta esa instancia", "pero ¿por qué? Si es la vida de su hijo", "bueno, pero el concepto de maternidad que usted tiene no es el que maneja la familia", ...y cómo ir planteando esas cosas. Y “¿por qué me dice esto a mi? Dígaselo a la madre", nos decía la doctora. Entonces hay que ir lidiando con este tipo de planteos. (TS género masculino, hospital de alta complejidad).

El valor que se le atribuye a la autonomía no se circunscribe a la posibilidad de tomar decisiones respecto de los abordajes sino también a la capacidad de encuadrar la tarea que los profesionales realizan, frente al resto del equipo de salud y directivos de la institución.

El gerente asistencial anterior, quien era un médico que se formó y estuvo siempre en el hospital, me permitió sacarle al servicio social todo lo que era de tilde administrativo. Me escuchó, hizo resoluciones, y bueno, pudimos dejar de hacer yo te diría un $80 \%$ de tareas que no nos corresponden. (TS de planta permanente, hospital de alta complejidad).

Desafiar los límites institucionales emerge como una tarea constante que genera cansancio laboral entre las y los trabajadores sociales. Sin embargo, una parte de ellos construye y jerarquiza la relativa autonomía y se asume como sujeto de su trabajo, sin victimizarse. Se sienten en condiciones, por formación y por tesón, “de definir direcciones 
para nuestra acción, definir prioridades y formas de encaminarlas" (Iamamoto y Sánchez, 2002, p. 99).

\subsection{Riesgos, conflictos y respuestas para enfrentarlos en los espacios laborales}

La mayor parte de los consultados se sienten expuestos a accidentes, a inseguridad y/o a violencia en el trabajo, en especial en la relación con los usuarios/as (63\%). No obstante, la sensación de exposición a ese y otros riesgos es, en general, moderada ${ }^{11}$.

A la pregunta sobre quién o quiénes resultaban más confiables para solucionar problemas en caso de sufrir riesgos o violencia laboral, los encuestados privilegiaron a sus propios compañeros de trabajo (60\%), y al Colegio de Profesionales de Trabajo Social de Mendoza (40\%). Apenas 20\% manifestó que recurriría al gremio de los trabajadores de salud.

Como cuando se indaga acerca de las oportunidades de empleo, la potencia de la red entre pares vuelve a ser la opción más confiable y la primera a la cual se acude frente a los conflictos. Los y las trabajadores sociales que aceptaron participar de esta investigación ${ }^{12}$ perciben a su red de contactos como una instancia de protección frente posibles amenazas.

\subsection{Diálogo social y relaciones laborales}

La participación de los trabajadores en el mundo laboral, donde existen diferentes tipos de negociaciones e intercambio de opiniones en relación con las condiciones laborales, es instancia de diálogo social. La posibilidad de establecer este tipo de diálogo que puede ser llevado a cabo por representantes elegidos colectivamente o también por interacción directa entre el trabajador y el empleador (Somavía, 2014) se midió en este trabajo a partir de los índices de afiliación sindical. El cuestionario arrojó que el 65\% se encuentra afiliado al gremio de la salud,13 mientras que una minoría (15\%) se declara dispuesta a afiliarse próximamente.

11 La formulación de la pregunta fue: En su trabajo diario en el hospital, ¿qué tan expuesto/a está a estos riesgos? Siendo las opciones de respuesta posible, que debían clasificarse como Muy expuesto/Medianamente expuesto/ Poco o nada expuesto/a: 1. Accidentes/inseguridad en entrevistas fuera del hospital; 2 . Accidentes/inseguridad en entrevistas en el hospital; 3. Trato violento por parte de los usuarios/as; 4. Falta de supervisión en situaciones conflictivas; 5. Trato irrespetuoso por parte de otros trabajadores.

12 Es probable que el resto no. Nos atrevemos a afirmar que la mayoría de los no respondentes vivió la invitación al estudio para reflexionar acerca de su trabajo como amenazante.

13 El gremio de los profesionales de la salud de Mendoza (AMPROS) agrupa a todos los profesionales del sistema público de salud, pero desde su creación, en 2005, hasta la actualidad es dirigido por médicos. 
El 100\% de los consultados están matriculados en el Colegio de Profesionales de Trabajo Social de Mendoza. En nuestro contexto, este indicador no da cuenta de la opción de agruparse para resolver colectivamente problemas de las relaciones laborales, ya que, por ley, la matriculación en la entidad profesional es una condición para trabajar en la administración pública provincial.

Respecto de la participación en la toma de decisiones institucionales que afectan el trabajo, que es una dimensión puesta en valor desde la óptica del trabajo decente, los entrevistados comparten que tienen escasa incidencia, si bien algunos advierten que tienen cierto margen para negociar con las autoridades del hospital, aunque esas negociaciones, por lo general, tienen lugar una vez que se consuman nuevos lineamientos de trabajo.

Se lanzó el registro único de salud, el RUS, y nosotros nos enteramos en el servicio ¿Qué pasó? "Esto lo empiezan a llevar desde hoy ustedes, la ficha", nos dijeron. Y ¿quién nos capacitó?, ¿cómo se hace esto? ... Mirá, mi jefa en ese momento lo aceptó y bueno, por ahí no le quedaba mucho por decir. Dijo: “Bueno, sí, lo vamos a hacer, pero nos vamos a dar unos días para ver cómo nos organizamos, en qué momento, en qué horario". (TS de planta permanente, hospital de alta complejidad).

Relatos de esta especie desnudan cierta contradicción entre la alta autonomía profesional con la que se dice que se trabaja y la baja participación en decisiones institucionales que afectan la tarea diaria. De alguna manera, los trabajadores sociales se perciben organizacionalmente invisibilizados; sienten que pueden hacer valer su criterio técnico siempre y cuando no colisione con las expectativas organizacionales. Esta situación, que también es señalada como factor de malestar laboral, sería parte de las "posiciones y juegos" que, en la expresión de Nucci et al., (2018, p. 23), los profesionales “deben disputar en el espacio político-institucional participando en las luchas discursivas acerca de las condiciones de vida de los sujetos y el lugar del Estado en la implementación de políticas públicas, a través de nuevas perspectivas".

\section{Conclusiones}

Consideramos que indagar en las condiciones laborales de los trabajadores sociales que se desempeñan en las organizaciones hospitalarias, a partir de dimensiones desarrolladas como la percepción de estabilidad presente y futura en el empleo, el trato respetuoso o las posibilidades de participar en la política organizacional, arroja información relevante para reflexionar acerca de la seguridad, la libertad y la dignidad con la que se trabaja. Valoramos también que los instrumentos utilizados en el trabajo de campo fueron adecuados para reconocer la particularidad con la cual se manifiestan dimensiones de Trabajo Social hospitalario sin generar interferencias en el espacio laboral y que la población consultada permitió profundizar en el constructo. 
Lo señalado no es menor si se tiene en cuenta "la baja jerarquía que el análisis de las condiciones de trabajo y salud de los trabajadores de la salud tiene en las producciones académicas y entre los propios trabajadores del sector hospitalario" (Organización Panamericana de la Salud, 2013, p. 198). Esta situación desentona con la alta proporción de trabajadores sociales insertos laboralmente en hospitales y con el valor simbólico que estos centros tienen para la sociedad.

En términos generales, los resultados obtenidos en el estudio concuerdan con otros realizados en otras jurisdicciones y en otras disciplinas relacionadas con la salud. En lo que hace a las singularidades de la profesión, resulta significativo identificar que, a pesar de las limitaciones, los riesgos y las tensiones puestas de manifiesto en esta investigación, los y las trabajadores sociales están altamente motivados para desempeñarse en el ámbito hospitalario, y que esa motivación se funda en la complejidad del proceso saludenfermedad-atención y en los desafíos que esa complejidad imprime a la práctica y a la formación profesional continua.

Como en otros estudios que ponen foco en las mujeres trabajadoras de la salud y en la relevancia de sus papeles en la producción de cuidados (Malleville, 2019; Ortega, 2019), y en sintonía con las experiencias del equipo de investigación, se constata que la feminización del Trabajo Social hospitalario agrega la tensión de la "doble presencia". Es decir, se está en el trabajo ocupándose de cuestiones familiares, y en el hogar, atendiendo situaciones laborales.

El análisis de los resultados muestra también que un alto porcentaje de trabajadores sociales experimenta situaciones de trato injusto en el día a día del trabajo, que por lo general se adjudican a las expectativas de respuesta rápida por parte de la administración y de los equipos médicos y, de modo más profundo, al modelo biologicista, individual y curativo que sigue prevaleciendo en los servicios de salud. Subyacería a los malentendidos y destratos interprofesionales la antigua noción del Trabajo Social como profesión subsidiaria, al servicio del control y la "normalización". La lucha cotidiana contra esa perspectiva genera confrontaciones y es vivida como causa central de desgaste laboral al interior de la disciplina, debido a la constante necesidad que refieren los trabajadores sociales consultados de tener que argumentar los alcances de las intervenciones frente al resto del equipo.

No obstante lo expuesto, se advierte también que los y las trabajadores sociales entienden que gozan de grados importantes de autonomía relativa que les permite abordar con libertad y criterio profesional las situaciones específicas que enfrentan. Esto no quita el reconocimiento de su baja injerencia en los espacios de toma de decisiones y en la formulación de las políticas organizacionales. Como mucho, sienten que pueden 
desplegar estrategias formales e informales para hacer frente al impacto de las decisiones sobre las cuales no han sido consultados.

En lo que se refiere a la protección en el trabajo, el Colegio Profesional aparece como espacio de escucha, de diálogo, en el que es posible revisar el sentido del trabajo y resolver los conflictos que conlleva. Esos conflictos se relacionan las más de las veces con la exposición a riesgos laborales, como accidentes en las entrevistas fuera y dentro del hospital, falta de supervisión en casos conflictivos y trato irrespetuoso por parte de otros trabajadores, entre otras situaciones que provocan déficit en el trabajo decente que emergieron en el estudio.

La indagación realizada posibilita afirmar que el concepto de trabajo decente ofrece potencialidades para ser aplicado de manera creativa en diferentes ámbitos de inserción disciplinar donde se desempeñan los y las trabajadores sociales. De la experiencia realizada se desprende la riqueza de utilizar un concepto flexible, que permite incorporar variables emergentes, como la feminización del trabajo hospitalario, la motivación para trabajar, la relevancia de ser valorados en los equipos de salud y las posibilidades y límites de la autonomía profesional.

En lo metodológico, se rescata haber podido constatar que todo recorrido investigativo conlleva una artesanía intelectual y ejercicios de reflexividad que se ven facilitados cuando no se trabaja en soledad. Someter al análisis y la discusión permanente los procedimientos, ponderar las consecuencias de modificar asunciones previas, y poder adaptarse a inquietudes de la población de estudio, sin tener que renunciar al propósito de lograr resultados válidos, es un aprendizaje clave en el propio proceso de formación profesional. En esta línea de pensamiento, resulta relevante compartir la trastienda de la investigación y dar cuenta de las singularidades del proceso seguido y aquí expuesto, dado el involucramiento del equipo de estudio con el problema a investigar.

Un reto no menor fue conseguir que, efectivamente, los y las colegas consultados respondieran a los cuestionarios enviados. Se hizo imprescindible generar estrategias de seguimiento, las que se materializaron como recordatorios e insistencias, junto a la continua garantía del anonimato de las respuestas. Estas dificultades, como se detalló en el apartado metodológico, no solo dilataron la obtención de datos, sino que terminaron modificando las técnicas del diseño original. El hecho de que esta situación haya generado en un comienzo cierto desconcierto en el equipo puede deberse al supuesto inicial de que trabajar con colegas de un mismo campo disciplinar, que sostiene principios de participación y reflexión crítica de las prácticas, podía desarrollarse de modo más fluido. Por el contrario, esta investigación transitó los mismos avatares que otras, a pesar de la relevancia que se esperaba que el tema tuviera para la población de estudio. 
Molina, Roslan-Angeloni, Correa y Varela

Las vicisitudes a las que nos expuso el problema abordado nos obligaron a reflexionar respecto de nuestras implicancias, a acercarnos y alejarnos de manera continua para poder observar y analizar el fenómeno con rigurosidad y no encandilarnos con la "ilusión de transparencia", sobre la que previenen Bourdieu y Wacquant (2008).

El desafío de agudizar la lectura de los datos emergentes a lo largo del proceso y el replanteo de estrategias, por parte del equipo de investigación, se vio favorecido con la incorporación de profesionales externos, quienes enriquecieron la labor desde la especificidad de su dominio disciplinar y le imprimieron objetividad al estudio.

Por último, se concluye que, como en este caso, cuando el equipo de investigación está altamente involucrado en el problema por investigar, acercarse a él a partir de categorías ya probadas en otros estudios y recurrir a terceros investigadores pone límites a la subjetividad. Más allá de las vicisitudes expuestas, puede resultar ventajoso conocer de antemano el ámbito a estudiar y hacer uso de los capitales simbólicos y sociales disponibles para analizar en profundidad problemas con sentido para el colectivo profesional.

\section{Referencias bibliográficas}

Acevedo, G., Farías, A., Sánchez, J., Astegiano, C., y Fernández, A. (2012). Condiciones de trabajo del equipo de salud en centros de atención primaria desde la perspectiva del Trabajo Decente. Revista Argentina de Salud Pública, 3(12), 15-22.

Amezcua, M. y Gálvez, A. (2002). Los modos de análisis en investigación cualitativa en salud: perspectiva crítica y reflexiones en voz alta. Revista Española de Salud Pública, 76(5), 423-436.

Anker, R., Chernyshev, I., Egger, P., Mehran, F. y Ritter, J. (2003). La medición del Trabajo Decente con indicadores estadísticos. Revista Internacional del Trabajo, 122(2), 161-195.

Aspiazu, E. (2016). Heterogeneidad y desigualdades de género en el sector salud: entre las estadísticas y las percepciones sobre las condiciones de trabajo. Revista Pilquén, 19(1), 55-64.

Aspiazu, E. (2017). Las condiciones laborales de las y los enfermeros en Argentina: entre la profesionalización y la precariedad del cuidado en la salud. Revista Trabajo y Sociedad, (28), 11-35.

Bourdieu P., y Wacquant, I. (2008). Una invitación a la sociología reflexiva. Buenos Aires: Siglo XXI Editores.

Brito-Quintana, P. (2000). Impacto de las reformas del sector de la salud sobre los recursos humanos y la gestión laboral. Revista Panamericana de Salud Pública, 8(1-2): 43-54.

Cazzaniga, S. (2007). Hilos y nudos. La formación, la intervención y lo político en Trabajo Social. Buenos Aires, Argentina: Editorial Espacio. 
Gálvez-Santillán, E. (2016). Trabajo decente. Una comparación entre los médicos del sector salud en Nuevo León y el contexto nacional. Trayectorias, 18(42), 94-108.

Gilson, L. (Ed.). (2012). Health policy and systems research: A methodology reader. Geneve: Alliance for Health Policy and Systems Research. World Health Organization. Recuperado de http:/ / www.who.int/alliance-hpsr/resources/alliancehpsr_reader.pdf.

Homedes, N., \& Ugalde, A. (2005). Human resources: the Cinderella of health sector reform in Latin America. Human Resources for Health 3(1). doi: 10.1186/1478-4491-3-1.

Iamamoto, M., y Sánchez, D. (2002). Intervención profesional frente a la actual cuestión social. En S. Severini (Coord.), Trabajo Social y mundialización. Etiquetar desechables o promover inclusión (pp. 91-126). Buenos Aires, Argentina: Editorial Espacio.

Lanari, M. (2010). Realidad y percepción del déficit de trabajo decente. El caso de los médicos que desempeñan sus tareas en hospitales de Mar del Plata. En L.A. Aguirre, A. Andreoni, R.M. Armendáriz y M. Arocena. (Eds.), Concurso bicentenario de la patria. El estado de la clase trabajadora en la provincia de Buenos Aires. La Plata: Ministerio de Trabajo de la Provincia de Buenos Aires. Recuperado de http://nulan.mdp.edu.ar/1039/.

Laurell, A. C. (2014). Contradicciones en salud: sobre acumulación y legitimidad en los gobiernos neoliberales y sociales de derecho en América Latina. Saúde em debate, 38(103), 853-871. doi: 10.5935/0103-1104.20140088.

Malleville, S. (2019). ¿Calidad en la atención, calidad en el empleo? Las fuentes de intensificación laboral en ocupaciones de cuidado, análisis de dos establecimientos de salud privada. Revista Trabalho (En)Cena, 4(esp.), 89-109. doi: $\underline{10.20873 / 2526-}$ 1487V4NEspecialP89.

Marradi, A. (2002). Método como arte. Papers. Revista de sociología, 67, 107-127.

Molina, C. y Tobar, F. (2018). ¿Qué significa Neoliberalismo en salud? RevIISE - Revista de Ciencias Sociales y Humanas, 12(12), 65-73. Recuperado de: http://www.ojs.unsj.edu.ar/index.php/reviise/article/view/272/pdf.

Neffa, J. C. (1995). Las condiciones y medio ambiente de trabajo (CyMAT). Presentación de la concepción dominante y de una visión alternativa. Buenos Aires, Argentina: Programa de investigaciones económicas sobre tecnología, trabajo y empleo. Consejo Nacional de Investigaciones Científicas y Tecnológicas. Recuperado de http://www.relats.org/documentos/SST.General.Neffa.CYMAT1995.pdf.

Neffa, J.C. y Henry, M.L. (Coords.). (2017). ¿Quién cuida a los que cuidan? Los riesgos psicosociales en el trabajo en los establecimientos privados de salud. La Plata, Argentina: Universidad Nacional de La Plata. Facultad de Ciencias Económicas. Recuperado de http://sedici.unlp.edu.ar/handle/10915/72269.

Nucci, N., Crosetto, R., Bilavcik, C., y Miani, A. (2018). La intervención de Trabajo Social en el campo de la salud pública. Conciencia Social. Revista digital de Trabajo Social, 1(2): 10-28. Recuperado de https://revistas.unc.edu.ar/index.php/ConCienciaSocial/article/view/19938.

Organización Panamericana de la Salud. (2013). La salud de los trabajadores de la salud. Trabajo, empleo, organización y vida institucional en hospitales públicos del aglomerado 
Gran Buenos Aires, Argentina, 2010-2012. Buenos Aires, Argentina: OPS/OMS. Recuperado de https://www.paho.org/arg/images/gallery/pub69.pdf?ua=1.

Ortega, J. (2019). Trabajos de(s) cuidados: reformas neoliberales, feminización y precarización de las condiciones de trabajo en enfermería. Athenea Digital, 19(3), e2333. doi: $10.5565 / \mathrm{rev} /$ athenea.2333.

Quintana-Zavala, M., Valenzuela-Suazo, S., y Paravic-Klijn, T. (2014). Enfermería desde la perspectiva del Trabajo Decente. Enfermería Global 13(33), 302-309.

Rovere, M., y Abramzón, M. (2005). Recursos Humanos en Salud. Bases para una agenda impostergable. Recuperado de https://docplayer.es/5393951-Recursos-humanos-ensalud-bases-para-una-agenda-impostergable-mario-rovere-monica-abramzon.html.

Salas-Zapata, W., Ríos-Osorio, L., Gómez-Arias, R., y Álvarez del Castillo, X. (2012). Paradigmas en el análisis de políticas públicas de salud: limitaciones y desafíos. Revista Panamericana de Salud Pública 32(1), 77-81.

Sánchez, J. (2010). La situación de la fuerza laboral del sector salud de Argentina bajo la perspectiva del trabajo decente (Tesis de maestría). Universidad Nacional de Córdoba, Facultad de Ciencias Médicas, Escuela de Salud Pública. Córdoba, Argentina.

Somavía, J. (2014). El trabajo Decente. Una lucha por la dignidad humana. Santiago, Chile: Organización Internacional del Trabajo. Recuperado de https://www.ilo.org/wcmsp5/groups/public/---americas/---ro-lima/---srosantiago/documents/publication/wcms_380833.pdf.

Sousa-Minayo de, M. C. (2009). La artesanía de la investigación cualitativa. Buenos Aires: Lugar Editorial.

Vasilachis, I. (1992). Métodos Cualitativos I. Los problemas teórico-epistemológicos. Buenos Aires, Argentina: Centro Editor de América Latina. 


\section{OTROS ARTÍCULOS DE PROSPECTIVA No. 30 DE 2020}

\section{EDITORIAL}

Coherencia, integridad y vida cotidiana

Luz Mary Sánchez-Rengifo

\section{ARTÍCULOS}

Trabajo Social y medios de comunicación: perspectivas y posibilidades de encuentro Social

Mercedes Muriel-Saiz

Maribel Martín-Estalayo

Seguridad, afectos y familias. Obstáculos en el proceso de reintegración de personas desmovilizadas de grupos

armados en Santander, Colombia

Jakeline Vargas-Parra

Ángela María Díaz-Pérez

Priscyll Anctil-Avoine

Reflexividad sobre la intervención profesional en duelo con población afectada por el conflicto armado en Colombia

María Cénide Escobar-Serrano

Maritza Charry-Higuera

Natalia Ramírez-Moncada

Ser mujer indígena, náhuatl, casada, migrante, sin trabajo remunerado: una realidad en los albergues jornaleros agrícolas en Colima, México

Nancy Elizabeth Molina-Rodríguez

Tipos y manifestaciones de la violencia de género: una visibilización a partir de relatos de mujeres víctimas en Soacha, Colombia

Diana Carolina Tibaná-Ríos

Diana Alejandra Arciniegas-Ramírez

Ingrid Julieth Delgado-Hernández

Análisis de necesidades en familias monoparentales con jefatura femenina usuarias de servicios sociales de atención primaria en España

Celia María Fernández-Martínez

Manuela Avilés-Hernández

Experiencias de intervención de trabajadoras sociales con trabajadores sexuales masculinos en Bogotá, Colombia Johan Arturo Barrera-Castellanos
Condiciones laborales de trabajadores sociales en hospitales públicos en la provincia de Mendoza,

Argentina

Cecilia Amalia Molina

Yanina Noemi Roslan-Angeloni

Analía Graciela Correa

Viviana Elena Varela

Gubernamentalidad neoliberal: miradas desde las intervenciones del Trabajo Social en el Gran La Plata, Argentina

Paula Mara Danel

Marcela Claudia Velurtas

Agustina María Favero-Avico

Educación superior en Trabajo Social en Chile y formación para la intervención en situación de calle. Desafios desde la evaluación que interventores hacen de sus procesos de práctica pre-profesional

Carlos Alejandro Andrade-Guzmán

Ignacio Andrés Eissmann-Araya

Educación en Derechos Humanos para el Trabajo Social en Chile: una mirada desde los estándares

internacionales

Lury Soledad Reyes-Pérez

Vivianne Soledad Hasse-Riquelme

Luis Marcelo Silva-Burgos

Arriesgar y preservar la vida: derechos humanos, conflicto sociopolítico armado y Trabajo Social en Colombia

Cristian Sebastián Castaño-Orozco

Ricardo Patiño-Martínez

\section{IN MEMORIAM}

Cristina Bautista Taquinás. Mujer indígena Nasa, Trabajadora Social, lideresa del norte del Cauca, Colombia

Alba Nubia Rodríguez-Pizarro

Lady Johanna Betancourt-Maldonado
ARTISTA INVITADO
MALA JUNTA KLAN
Alejandra Gutiérrez-Cárdenas

\section{PROSPECTIVA}

